Article

\title{
Investigations into the Potential Abrasive Release of Nanomaterials due to Material Stress Conditions-Part B: Silver, Titanium Nitride, and Laponite Nanoparticles in Plastic Composites
}

\author{
Johannes Bott * and Roland Franz \\ Department of Product Safety and Analytics, Fraunhofer Institute for Process Engineering and Packaging (IVV), \\ 85354 Freising, Germany; roland.franz@ivv.fraunhofer.de \\ * Correspondence: johannes.bott@ivv.fraunhofer.de; Tel.: +49-8161-491-753
}

Received: 7 December 2018; Accepted: 20 December 2018; Published: 9 January 2019

\begin{abstract}
Three plastic nanocomposites containing the nanomaterials silver, titanium nitride, and laponite were investigated on the potential to release nanoparticulates under stress conditions into food simulants. Nanocomposites were exposed to thermal, chemical, and mechanical stress followed by mechanical abrasion of their surface. Particle sensitive asymmetric flow field-flow fractionation (AF4) with multi-angle laser light scattering (MALLS) as well as inductively coupled plasma mass spectrometry (ICP-MS) detection was used to detect and quantify the respective nanoparticulates. The results of this study demonstrate that even under dynamic stress conditions nanoparticulates are not released from the nanocomposites into food.
\end{abstract}

Keywords: nanosilver; titanium nitride; laponite; nanomaterial; nanoparticle; release; migration; mechanical stress test; nanocomposite; food contact

\section{Introduction}

This article is the second in a series of two dealing with the potential release of nanomaterials after intense mechanical-, thermal-, and chemically-induced stress conditions. Nanomaterials are used in consumer products like food packaging articles which require profound knowledge on the potential release of nanomaterials into foods for proper risk assessment. In our previous works, based on comprehensive migration testing using plastics nanocomposites with incorporated silver nanoparticles (Ag-NPs) [1], nanotitanium nitride (TiN) [2], and nanolaponite [3], we could not find any detectable migration and together with theoretical considerations we concluded that migration of nanoparticles from plastic food packaging materials in general is not possible according to Fickian Law of diffusion as long as the particles are larger than 3-4 $\mathrm{nm}$ (in case of polyolefins) or 1-2 $\mathrm{nm}$ (for PET and similar polymers) in diameter [4] and when the nanomaterial is fully covered with polymer (i.e., does not stick out of the polymer). Since such small nanomaterial sizes are not used in food contact nanocomposites exposure of the consumer due to diffusion-controlled migration of nanomaterials can be excluded.

In a number of publications positive results for nanoparticle migration were reported. A closer view into these publications revealed that the migration tests were done using cut strips or pieces of polymer films or articles or using very aggressive food simulants which destroyed the polymer [5]. From these findings and from the generally raised question whether under stress conditions in use nanomaterials could be released from the food contact surface of nanocomposites, the motivation for this project was taken to explore whether such mechanical release of nanomaterials may occur and if yes, how it could be tested. In Part A (reference to: Appl. Sci. 2018, 8, Bott, J., Franz, R., "Investigations into the potential abrasive release of nanomaterials due to material stress conditions 
- Part A: carbon black nanoparticulates in plastic and rubber composites") of this series a possible experimental design to test release of nanomaterials after mechanical-, thermal-, and chemical-induced stress was proposed and already tested on nanocomposites containing carbon black. It was demonstrated that carbon black will not be released as long it is fully incorporated into the polymer. In Part B of this series, the same experimental design and abrasion test will now be used on Ag, TiN, and laponite nanocomposites.

\section{Materials and Methods}

\subsection{Materials}

In our previous studies the diffusion (in polymer) controlled migration potential of TiN-NP [2], Ag-NP [1], and Laponite [3] from LD-PE-based composites was investigated. The same nanocomposites were now used to investigate the potential mechanical release of nanoparticles. An overview of the used nanocomposites is given in Table 1. All nanocomposites were rolled to films with a width of $21 \mathrm{~cm}$ and a thickness of $60 \mu \mathrm{m}$. A short summary of the nanocomposites properties is given in the next subsections including transmission electron microscopy (TEM) images. TEM images of the polymeric films were prepared by psi cube, Germany. With this technique the distribution and size characteristics of the laponite in the polymer was visualized. For sample preparation the polymeric film were subjected to cryo-ultrathin-sectioning using a diamond knife.

Table 1. LDPE-based nanocomposites used as test materials.

\begin{tabular}{ccc}
\hline Nanomaterial & Polymer Matrix & Nanomaterial Loading \\
\hline $\begin{array}{c}\text { Silver Nanoparticles } \\
(\text { Ag-NP) } \\
\text { Titaniumnitride } \\
(\text { TiN) } \\
\begin{array}{c}\text { Laponite } \\
\text { (LAP) }\end{array}\end{array}$ LD-PE & $250 \mathrm{mg} / \mathrm{kg}$ \\
& LD-PE & $1000 \mathrm{mg} / \mathrm{kg}$ \\
\hline
\end{tabular}

\subsubsection{Ag-NP Nanocomposites}

In plastic food packaging materials Ag-NPs are primarily used as an antimicrobial agent. Ag-NPs are fine particles of elemental silver that exist as discrete entities in the polymer matrix. In contrast to many other nanomaterials Ag-NPs do not exhibit complex structures (Figure 1), like highly branched aggregates (e.g., TiN), which results in higher mobility compared to other nanomaterials can be assumed. However, in our previous study it was already demonstrated that Ag-NPs do not migrate out of a polymer matrix when in contact with food/food simulants. In this study Ag-NPs nanocomposites were chosen due to the potential use of high specific detection techniques like ICP-MS. 


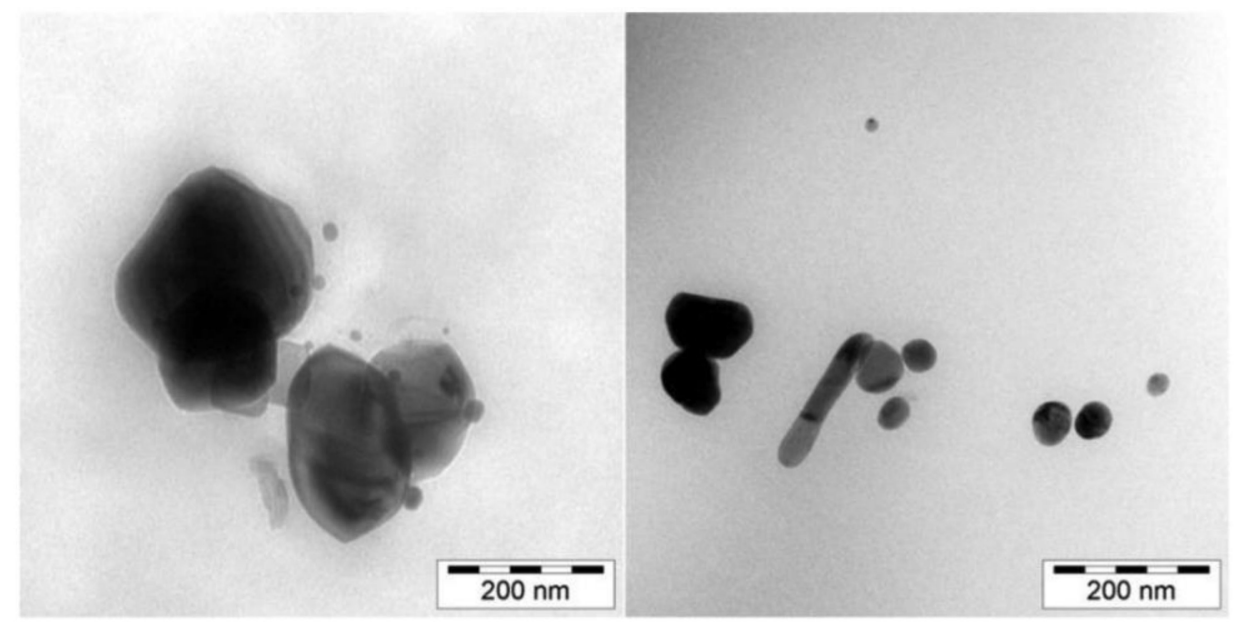

Figure 1. Transmission electron microscopy (TEM) images of an AgNPs-nanocomposite.

\subsubsection{TiN Nanocomposites}

Nanosized TiN is used as a so-called reheat additive in the production of bottles made of polyethylene terephthalate (PET). In our previous study the non-migratability of TiN-NPs from a LDPE-based nanocomposite was already demonstrated. From that study the nanocomposites were used to examine a release of TiN-NPs after mechanical stressing of the test samples. Due to the elemental composition of TiN element specific techniques like (ICP-MS) can be used to analyze for titanium very specifically at low detection limits. Transmission electron microscopy (TEM) images (Figure 2) of the TiN-nanocomposite showed a homogeneous distribution of NPs in the LDPE matrix. Thereby, the aggregates consist of almost spherical primary particles with an average size of $20 \mathrm{~nm}$ that are fused to irregular formed aggregates of an average size of $100-500 \mathrm{~nm}$.

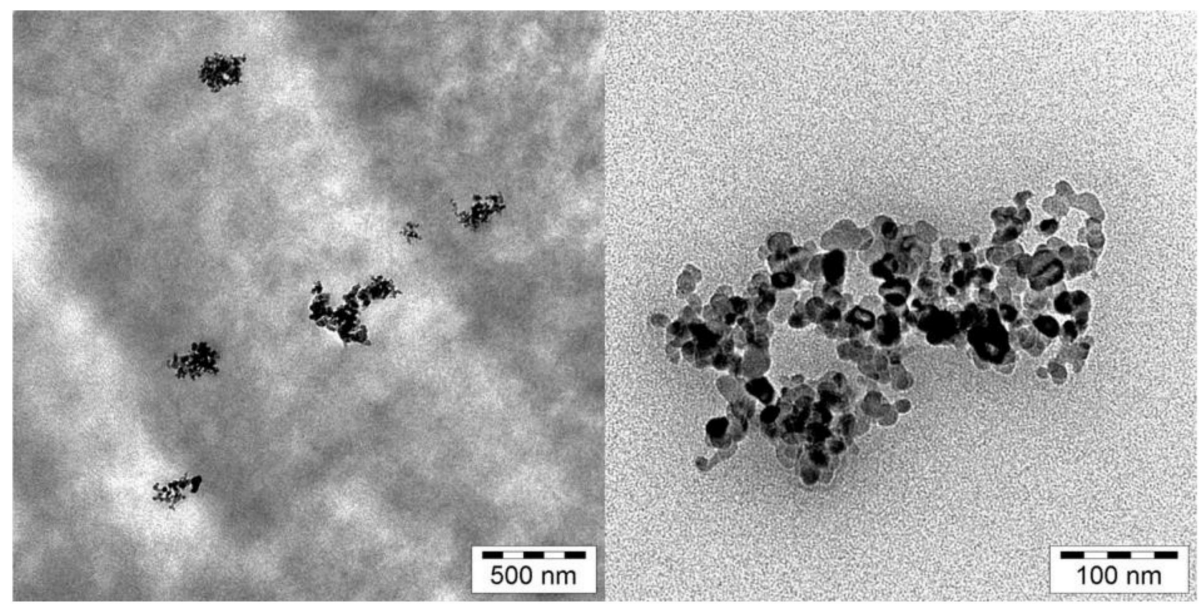

Figure 2. TEM images of a TiN/LDPE-nanocomposite.

\subsubsection{LAP Nanocomposites}

Laponite is a synthetic colloidal layered silicate which is composed of disc-shaped crystals in the nanoscale size region. In recent studies it was shown that the physicochemical polymer properties of biopolymers based on pectin [6] and cellulose ethers [7] could be enhanced by the incorporation of laponite. Laponite crystals are of approx. $1 \mathrm{~nm}$ in thickness and have diameters of approx. $25 \mathrm{~nm}$. Thus, the primary structure (i.e., the disc-shaped crystals) is significant smaller than it is in naturally occurring clay minerals, like bentonite, and for this reason laponite exhibits a higher potential to be released from a polymer matrix than other clay minerals. A visualization of the laponite particles as present in the used nanocomposite is given in Figure 3. The platelets within the laponite stack are rather 
oriented randomly than parallel to each other. At the border region of the Laponite aggregates single platelets can be found. Typical aggregates found in the laponite-nanocomposites exhibit diameter of approx. 50-200 nm whilst single laponite platelets show diameters of approx. $20-30 \mathrm{~nm}$ with a thickness of $1 \mathrm{~nm}$.

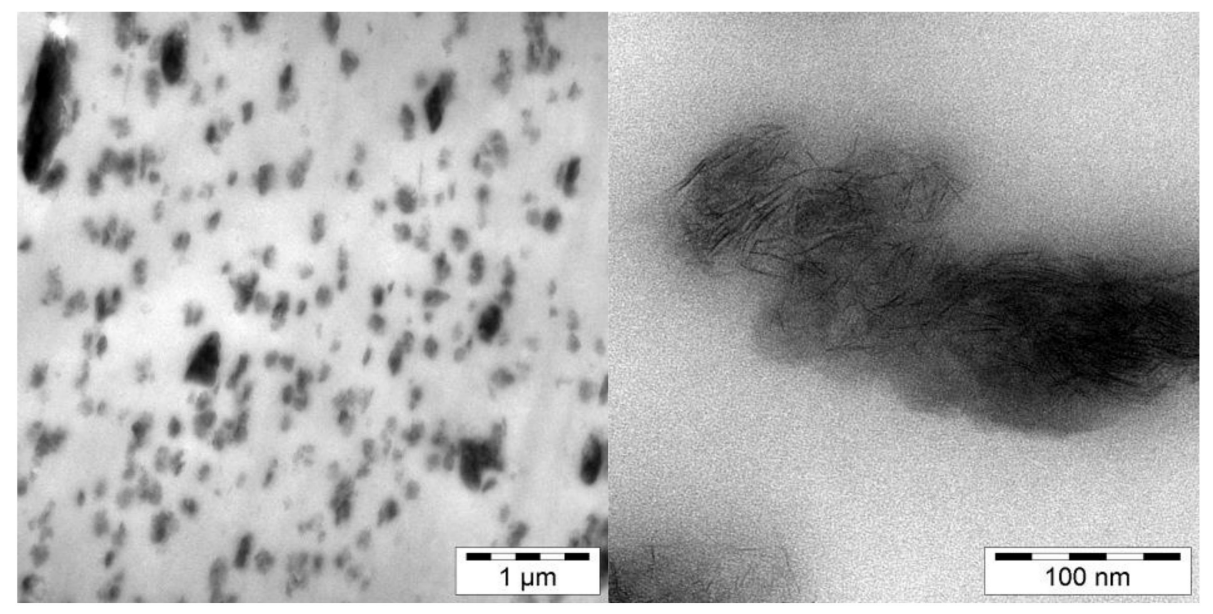

Figure 3. TEM images of a laponite-nanocomposite.

\subsection{Nanorelease Study}

The mechanical stress test of this study aimed to stress the surface of the nanocomposites and to evaluate whether release of nanomaterials (nanomaterials) under stress conditions was possible. The setup of the stress test must therefore take influence on the surface of the nanocomposite whilst at the same time released nanomaterials must be picked up to be detected by following analytical techniques. The idea was to use an abrasive substance that scrubs the nanocomposites surface. In a further step the abrasive substance and the abrasion itself were collected completely and analysed by suitable techniques on the presence of nanomaterials. The basic setup of the nanorelease test is schematically displayed and described more in detail in part A of this series of publications.

\subsubsection{Basic Material Stressing-Abrasion Test}

Nanocomposites were clamped in glass Petri dishes and loaded with $1.0 \mathrm{~g}$ of an abrasive substance (i.e., salt or quartz sand). Depending on the chemical nature of the nanomaterial and the subsequent analytical technique used either quartz sand (Büchi Labortechnik GmbH, Germany) or salt (sodium chloride p.A., Sigma Aldrich Chemie $\mathrm{GmbH}$, Germany) were used as a dry abrasive simulant. The cells were composed of a smaller petri dish with an inner diameter of $90 \mathrm{~mm}$ and a larger petri dish with an inner diameter of $95 \mathrm{~mm}$. In a first step, the smaller cell was loaded with the dry food simulant and the nanocomposite was loosely placed on the dish. In a second step the larger cell was put over the smaller cell with the nanocomposite film in between. This way the nanocomposite was clamped in the cells with an even surface (Figure 4). Furthermore, the nanocomposite itself acted as a sealant between the cells to prevent loss of dry simulant and abrasion. By turning the cells upside down (small cell on top) the dry food simulant was then brought in contact with the nanocomposite. The surface area of the nanocomposite that was in contact with the dry food simulant was given by the inner diameter of the smaller cell $(90 \mathrm{~mm})$, which was $0.64 \mathrm{dm}^{2}$.

The abrasion test was started by placing the cells on an orbital shaker (GFL 3017, Gesellschaft für Labortechnik, Germany). The rotating movement of the cells caused the abrasive substance to homogeneously scrub over the nanocomposite surface. The intensity of the stress test could be varied by duration and the frequency of the shaking as well as by the amount of abrasive substance. 


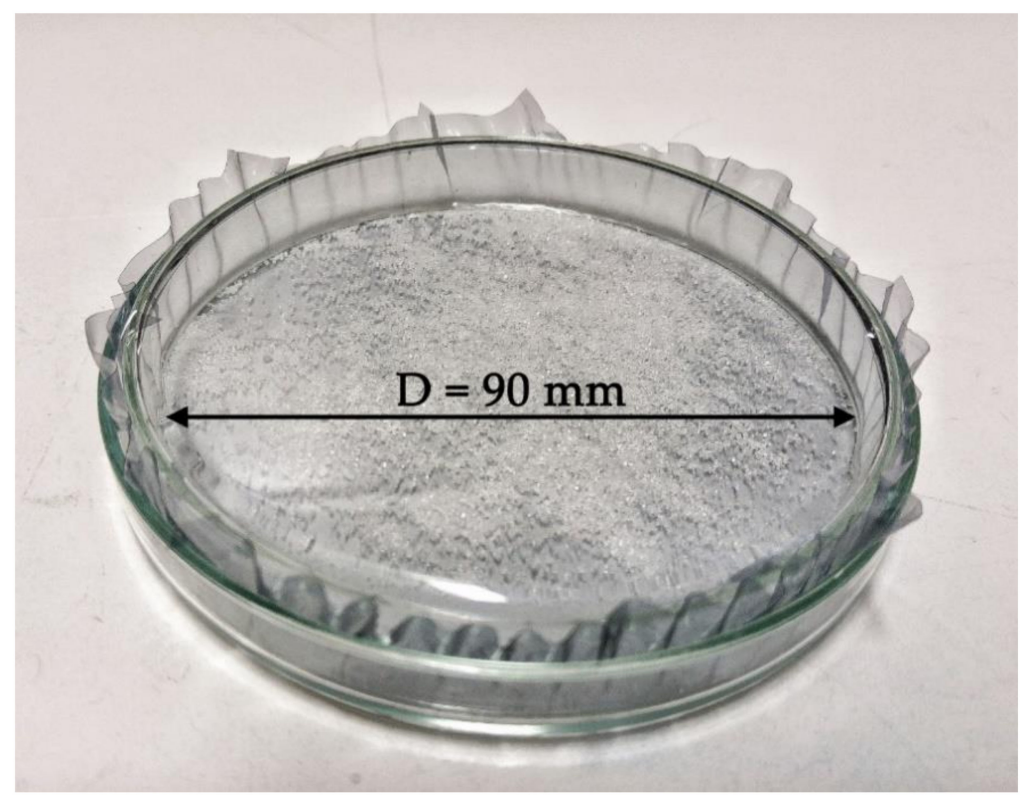

Figure 4. Abrasion cell loaded with a TiN/LDPE-nanocomposite and sodium chloride as abrasive substance.

For the abrasion test all cells were equipped with the nanocomposites as described above. In practice it turned out that best results can be achieved when the dry food simulants are stored in a desiccator before testing, to prevent adhesion on the nanocomposites surface caused by humidity.

The speed of the laboratory shaker was set to $275 \mathrm{rpm}$. This speed caused a flat distribution of the dry simulant on the nanocomposite, whereby the surface was always nearly completely covered. Also, at $275 \mathrm{rpm}$ the simulant showed a homogeneous rotating movement over the nanocomposites' surface, meaning that all parts of the nanocomposite were abraded evenly. For the surface abrasion test all samples were abraded with the dry food simulant for $60 \mathrm{~min}$ (without prestress conditions) or $30 \mathrm{~min}$ (with prestress conditions applied).

\subsubsection{Additional Material Stressing_-"Prestress Conditions"}

As described in part A of our other article prestress conditions can be applied to nanocomposites in advance to gather additional stress conditions that might appear in practice. In real life, the food contact material could be exposed to other mechanical impacts, thermal cycles or aggressive chemicals which could weaken the polymer and thus facilitate the release of nanomaterials after abrasion. Such potential stress situations were simulated in this study as follows.

- Thermal stress—heating:

Stripes of $50 \mathrm{~cm}$ of the nanocomposites were loosely folded, placed in closed foil pans, and stored in a temperature controlled oven for $24 \mathrm{~h}$ at $100{ }^{\circ} \mathrm{C}$. After cooling down to room temperature all samples were placed in abrasion cells and subjected to the abrasion test for $30 \mathrm{~min}$.

- Thermal stress—freezing:

Stripes of $50 \mathrm{~cm}$ of the nanocomposites were loosely folded, placed in closed foil pans and stored in a temperature controlled freezer for $24 \mathrm{~h}$ at $-50{ }^{\circ} \mathrm{C}$. After warming up in a desiccator (to prevent condensate formation) all samples were placed in abrasion cells and subjected to the abrasion test for $30 \mathrm{~min}$.

- Solvent based stress—swelling: 
Stripes of $20 \mathrm{~cm}$ of the nanocomposites were fan-folded, placed in stainless steel cells and filled with $100 \mathrm{~mL}$ isooctane, whereby the test films were completely immersed in the simulant. The cells were closed and sealed with a PTFE ring and stored in a temperature controlled oven for $24 \mathrm{~h}$ at $40^{\circ} \mathrm{C}$. Prior to mechanical stressing all samples was stored for $12 \mathrm{~h}$ under a laboratory hood to evaporate any remaining solvent. All samples were placed in abrasion cells and subjected to the abrasion test for $30 \mathrm{~min}$.

- Mechanical stress—stretching:

Cut-outs of $200 \times 140 \mathrm{~mm}$ of the nanocomposites were clamped in a universal tensile strength testing apparatus equipped with a traverse path sensor. The nanocomposites were stretched lengthwise for $100 \mathrm{~mm}$. After stretching the nanocomposites showed slight deformation. LDPE plates were slightly longer (approx. $10 \mathrm{~mm}$ ) and showed a slightly rippled surface. From all test samples circular cut-outs from the center of the stressed films were prepared and placed in the abrasion cells for abrasion testing. Abrasion testing was performed for $30 \mathrm{~min}$.

\subsection{Analytical Set-Up for the Detection of Released Nanomaterials in the Abrasion}

- Silver nanocomposites

Silver is an element that can be measured at very low detection limits by inductively-coupled plasma mass spectrometry (ICP-MS). For the detection of silver in samples taken from the abrasion test the instrument was calibrated using Merck VI ICP Multielement standard solution (Merck KGaA, Darmstadt, Germany). From this stock solution a serial dilution with 0.0 ( $3 \%$ nitric acid blank),0.5, 1.0, $1.5,2.0,2.5,5.0,10.0$, and $25.0 \mathrm{ng} / \mathrm{mL}$ silver in $3 \%$ nitric acid was prepared. The standard with the lowest concentration of silver $(0.5 \mathrm{ng} / \mathrm{mL})$ could still be distinguished from the blank and standards with higher silver concentrations. Thus, $0.5 \mathrm{ng} / \mathrm{mL}$ was set as the limit of detection of the device for silver. Quartz sand was used as an abrasive substance for silver nanocomposites because no interferences between solubilized silver particles and other ions can be expected. Stability tests were performed to evaluate whether loss of sample might adhere due to adsorption of silver on the surface of the sand particles. For this $1.0 \mathrm{~g}$ quartz sand was given to $20 \mathrm{~mL}$ of $10.0 \mathrm{ng} / \mathrm{mL}$ silver in $3 \%$ nitric acid solution and shaken for $5 \mathrm{~min}$. The supernatant was analyzed by ICP-MS and compared to a $10.0 \mathrm{ng} / \mathrm{mL}$ silver standard without sand. With a stability rate of $102.7 \%$ silver could be fully recovered.

- $\quad$ TiN nanocomposites

Proof of TiN NPs in the abrasion was intended to be performed by ICP-MS measurements of Ti. For this the ICP-MS was calibrated externally using a Ti standard solution (Merck KGaA, Darmstadt, Germany, Matrix: $3 \%$ nitric acid $\left(\mathrm{HNO}_{3}\right)$ ). From this stock solution a serial dilution with 0.0 ( $3 \%$ nitric acid blank), 1.0, 5.0, 10.0, 15.0, and $20.0 \mathrm{ng} / \mathrm{mL}$ titanium in $3 \%$ nitric acid was prepared and used for calibration of the ICP-MS. The standard with the lowest concentration of titanium $(1.0 \mathrm{ng} / \mathrm{mL})$ could still be distinguished from the blank and standards with higher titanium concentrations. Thus, $1.0 \mathrm{ng} / \mathrm{mL}$ was set as the limit of detection of the device for titanium. $\mathrm{NaCl}$ was tested on its suitability as a dry abrasive simulant in the abrasion test. For this, $\mathrm{NaCl}$ was tested on impacts of the Ti recovery rate. The Ti standards $(5 \mathrm{ng} / \mathrm{mL})$ in a $\mathrm{NaCl} / \mathrm{HNO}_{3}$ matrix $\left(1.0 \mathrm{~g} \mathrm{NaCl}\right.$ per $\left.20 \mathrm{~mL} \mathrm{HNO}_{3}(3 \%)\right)$ were compared to a $5 \mathrm{ng} / \mathrm{mL}$ Ti standard in $3 \% \mathrm{HNO}_{3}$ matrix without $\mathrm{NaCl}$. It turned out that $79.6 \%$ (average value from samples prepared in triplicate) of Ti could be recovered.

- Laponite nanocomposites

In a previous study [3] it was demonstrated that AF4/MALLS was suitable for the characterization and quantification of laponite particles dispersed in an aqueous surfactant solution (2000 mg/L NovaChem). However, AF4/MALLS showed limitations caused by superimpositions in the fractogram when the sample matrix became too complex. With the empiric formula 
$\mathrm{Na}_{0.7}\left[\left(\mathrm{Si}_{8} \mathrm{Mg}_{5.5} \mathrm{Li}_{0.3}\right) \mathrm{O}_{20}(\mathrm{OH})_{4}\right]^{-0,7}$ laponite is mainly composed of ubiquitous elements that do not allow sensitive detection via element-specific ICP-MS measurements, due to an already high background of laponite relevant elements. However, a combination of both techniques (i.e., AF4 with MALLS and ICP-MS as detection system) would allow simultaneous screening for particulate structures with an elemental composition typical for laponite.

For calibration of the AF4/MALLS system laponite reference dispersions in NovaChem surfactant solution at concentrations 0.0 (NovaChem blank), 250, 500, 1000, 2000, and $2500 \mathrm{ng} / \mathrm{mL}$ were prepared. The standard with the lowest concentration of laponite $(250.0 \mathrm{ng} / \mathrm{mL})$ could still be distinguished from the blank and standards with higher laponite concentrations. Thus, $250.0 \mathrm{ng} / \mathrm{mL}$ was set as the limit of detection of the device for laponite. To determine a suitable dry food simulant test with quartz sand and $\mathrm{NaCl}$ in the surfactant solution were performed. One gram of dry simulant was weighed out into measuring vials and filled with $20 \mathrm{~mL}$ surfactant solution $(2000 \mathrm{mg} / \mathrm{L}$ NovaChem) used as dispersant for laponite. After the samples were shaken for $5 \mathrm{~min}$ the salt was completely dissolved whilst the sand caused a slight opalescent supernatant. In fact, injections of these samples showed that supernatants of samples prepared with sand caused superimpositions in the AF4 fractogram, whilst samples prepared with sand did not. Thus, $\mathrm{NaCl}$ was further tested on its ability to be used as a dry food simulant for laponite particles. Laponite dispersions were prepared for recovery experiments whereby a $2500 \mathrm{ng} / \mathrm{mL}$ Laponite dispersion with an additional $1.0 \mathrm{~g} \mathrm{NaCl}$ per $20 \mathrm{~mL}$ surfactant solution was measured against a $2500 \mathrm{ng} / \mathrm{mL}$ Laponite dispersion without salt in the surfactant solution. AF4/MALLS measurements demonstrated that approx. $65.3 \%$ of the laponite could be recovered as particles. The loss of signal intensity can be explained by the high ionic strength of the dispersion due to the high content of salt, which affects the dispersion stability of clay particles and might cause sedimentation of sample.

In regard to the later measurements of samples prepared from the abrasion test superimpositions might disturb an unambiguous detection of laponite in the AF4/MALLS fractogram. Thus, AF4/MALLS/ICP-MS measurements were performed to see whether via online detection of the laponite-specific element magnesium $(\mathrm{Mg})$ a better differentiation can be achieved. Injections of a $2 \mathrm{mg} / \mathrm{L}$ laponite dispersion caused a signal at the relevant elution times in the fractogram recorded with the MALLS detector (black curve, Figure 5) but also in the fractogram recorded via ICP-MS (red curve for Mg, Figure 5) whilst samples prepared from the dry simulant itself (i.e., salt in surfactant solution) did not cause a signal. These measurements showed that in case of release of laponite a signal in both detection modes, AF4/MALLS and AF4/ICP-MS will be recorded.

After completion of the abrasion test the sand or salt and abrasion was carefully transferred into $50 \mathrm{~mL}$ centrifugal vials. Abraded Nanocomposites were rinsed with the respective solution $\left(3 \% \mathrm{HNO}_{3}\right.$ in case of Ag-NPs or TiN nanocomposites or surfactant solution in case of laponite nanocomposites) whereby the rinsing fluid was collected into the centrifugal vials together with the abrasion and the sand or salt. All vials were the filled with the respective solution to the $20 \mathrm{~mL}$ mark and shaken on a laboratory shaker for $15 \mathrm{~min}$ to detach or disperse possibly released nanomaterials.

Instrumentation

AF4 measurements were carried out with an "AF2000 MT Series mid temperature" (Postnova Analytics, Landsberg, Germany). The system was equipped with a $500 \mu \mathrm{m}$ channel and a polyethersulfone membrane (cut-off: $10 \mathrm{kDa}$ ). Characterization of particles was performed online using a 21-angle-MALLS detector "PN3621" (Postnova Analytics, Landsberg, Germany). ICP-MS measurements were performed using a 7700 series ICP-MS system (Agilent Technologies, Santa Clara, CA, USA). The ICP-MS was equipped with an ASX-520 autosampler (Agilent Technologies, Santa Clara, CA, USA) and a micromist nebulizer (Agilent Technologies, Santa Clara, CA, USA). The system operated at $1550 \mathrm{~W}$ plasma power, $0.3 \mathrm{rps}$ peristaltic pump speed, and $15 \mathrm{l} / \mathrm{min}$ carrier gas flow rate (argon). 


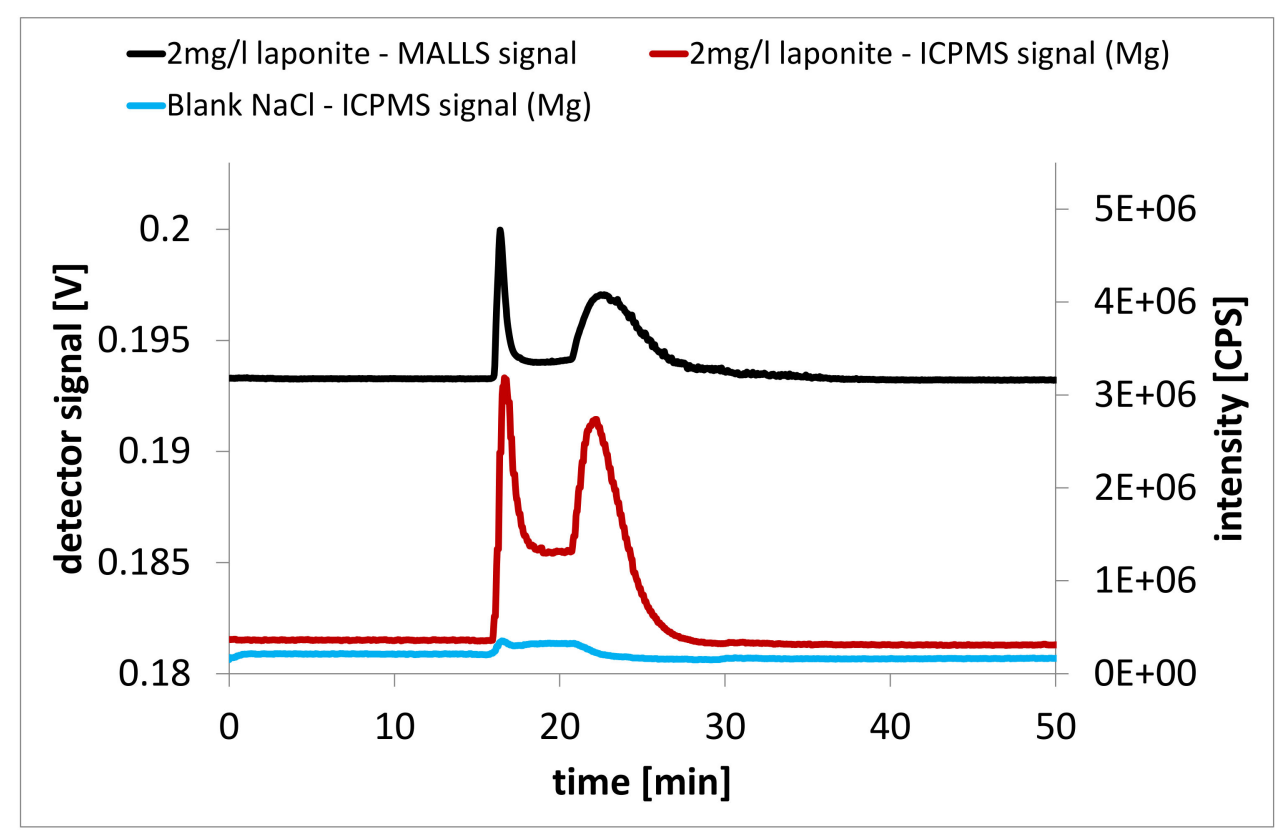

Figure 5. Comparison between AF4/MALLS and AF4/ICPMS measurements of a $2 \mathrm{mg} / \mathrm{L}$ laponite dispersion. Black is the signal recorded by the MALLS detector (caused by laponite particles) and red is the signal of the ICP-MS for $\mathrm{Mg}$ at identical elution times. Blue is the signal for $\mathrm{Mg}$ of the $\mathrm{NaCl}$ in $3 \%$ $\mathrm{HNO}_{3}$ blank (shifted by $-1.5 \times 10^{5}$ CPS for better differentiation).

\section{Results}

\subsection{Silver Nanocomposites}

AgNPs-nanocomposites were tested on release of silver after abrasion or after prestressing followed by abrasion (Table 2). All samples were prepared in triplicate and analyzed for silver via ICP-MS. From the LOD of the device $(0.5 \mathrm{ng} / \mathrm{mL})$ and with respect to the previously described set-up and validation results of the study, the overall method LOD was $16.0 \mathrm{ng} / \mathrm{dm}^{2}$. Validation measurements demonstrated that silver would have been detected if abraded. However, none of the abrasion samples caused a signal for silver above the method LOD.

Table 2. Results of the abrasion test performed with silver nanocomposites.

\begin{tabular}{ccc}
\hline Abrasion Test & Prestress Condition & $\begin{array}{c}\text { Ag in Abrasion } \\
{\left[\mathbf{n g} / \mathbf{d m}^{2}\right]}\end{array}$ \\
\hline $60 \mathrm{~min}$ at $275 \mathrm{rpm}$ & $\begin{array}{c}\text { none } \\
\text { "heating } \\
30 \mathrm{~min} \text { at } 275 \mathrm{rpm}\end{array}$ & $<16.0$ \\
$30 \mathrm{~min}$ at $275 \mathrm{rpm}$ & $\begin{array}{c}\text { "freezing } 100{ }^{\circ} \mathrm{C} \\
\text { "24 h at }-50^{\circ} \mathrm{C} \\
\text { "swelling" }\end{array}$ & $<16.0$ \\
$30 \mathrm{~min}$ at $275 \mathrm{rpm}$ & $24 \mathrm{~h}$ at $40^{\circ} \mathrm{C}$ in isooctane \\
"stretching" & $<16.0$ \\
$30 \mathrm{~min}$ at $275 \mathrm{rpm}$ & irreversible transformation & $<16.0$ \\
\hline
\end{tabular}

\subsection{Titanium Nitride Nanocomposites}

All samples taken from the abrasion test with and without prior pre-conditioning were prepared in triplicate and analyzed for release of titanium via ICP-MS. From the LOD of the device $(1.0 \mathrm{ng} / \mathrm{mL})$ and with respect to the previous described set-up and validation results of the study the overall method LOD was $39.3 \mathrm{ng} / \mathrm{dm}^{2}$ calculated as Ti or $50.8 \mathrm{ng} / \mathrm{dm}^{2}$ calculated as TiN, respectively. ICP-MS measurements showed that already the reference samples without TiN in the polymer caused a 
significant signal for titanium. Titanium is an ubiquitous element and high background concentrations of titanium were already known from our previous migration study [2]. In this study the background of Ti was approx. $0.065 \mu \mathrm{g} / \mathrm{dm}^{2}$. The abrasion prepared from test samples with $1000 \mathrm{mg} / \mathrm{kg}$ TiN in LDPE did not cause higher signals when the nanocomposites were stressed mechanically (abrasion only) or after additional freezing (Figure 6).

Compared to the reference samples without $\mathrm{TiN}$ in the polymer the other test samples that were additionally stressed caused a relative higher release of titanium of approx. $0.03 \mu \mathrm{g} / \mathrm{dm}^{2}$ (heating), $0.08 \mu \mathrm{g} / \mathrm{dm}^{2}$ (swelling) and $0.11 \mu \mathrm{g} / \mathrm{dm}^{2}$ (stretching).

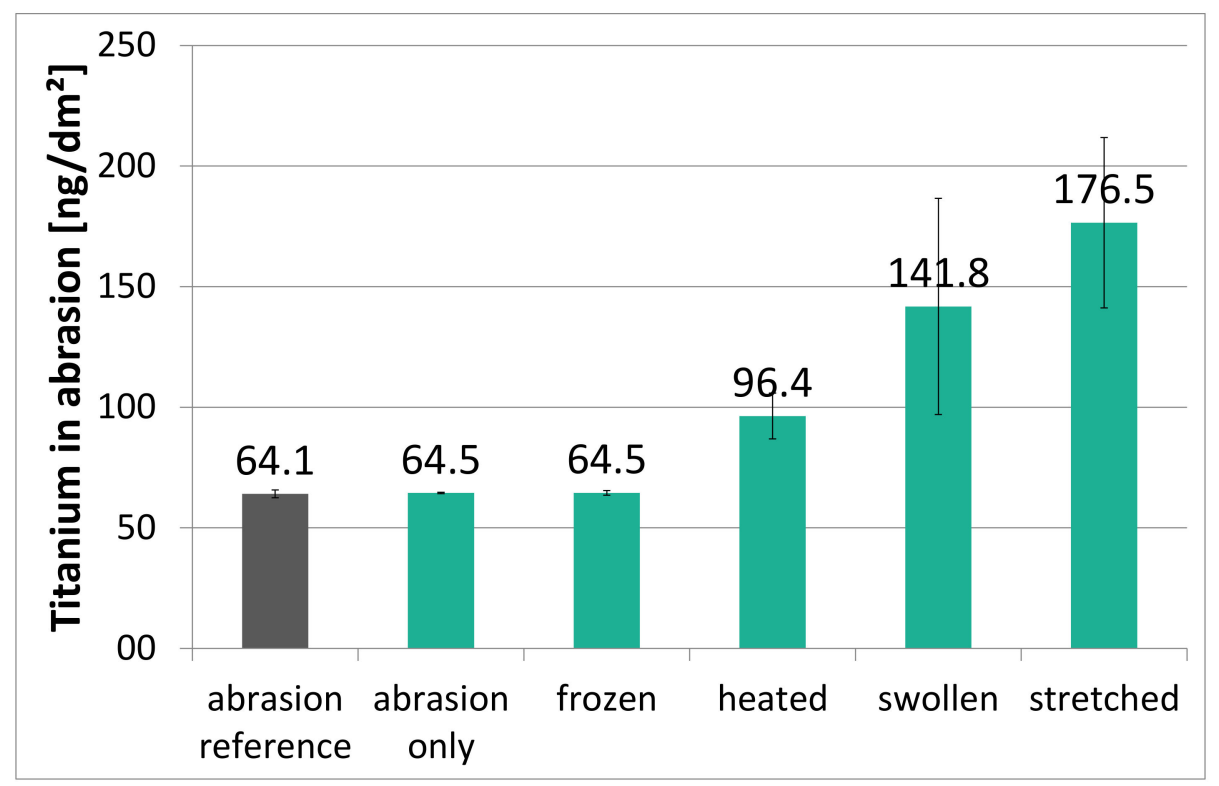

Figure 6. Titanium detected in the abrasion after stressing of the $1000 \mathrm{mg} / \mathrm{kg} \mathrm{TiN}$ in LDPE nanocomposite.

\subsection{Laponite Nanocomposites}

From the LOD of the device $(250.0 \mathrm{ng} / \mathrm{mL})$ and with respect to the previous described set-up and validation results of the study an overall method LOD of $12.0 \mu \mathrm{g} / \mathrm{dm}^{2}$ was derived. With respect to that method LOD no release of laponite was found from samples that were stressed by abrasion only or additionally by thermal stress (heating or freezing). Injections of such samples did not cause a signal at elution times relevant for laponite particles $(t=21-35 \mathrm{~min})$ the AF4 fractogram, which is exemplarily shown in Figure 7.

Injections of samples prepared from the abrasion of samples that were stored in isooctane previously and then abraded by the dry food simulant showed a signal at elution times relevant for laponite particles (Figure 8, black fractogram). As already mentioned above the MALLS detection is unspecific in case of coelution of laponite and any other matrix components. It is already known from the migration experiments that oligomers are extracted from the polymer when stored in isooctane. In this case no differentiation between laponite and coeluting oligomers is possible via the MALLS detection. However, the same sample did not cause a signal for Mg when measured by AF4/ICP-MS (Figure 8, red fractogram). From the validation experiments (see Figure 5) it is known that laponite particles cause a signal in both, the AF4/MALLS and even a higher signal in the AF4/ICP-MS fractogram. With a signal in the AF4/MALLS fractogram only, but no signal for Mg in the AF4/ICP-MS fractogram the presence of laponite particles can be excluded. 


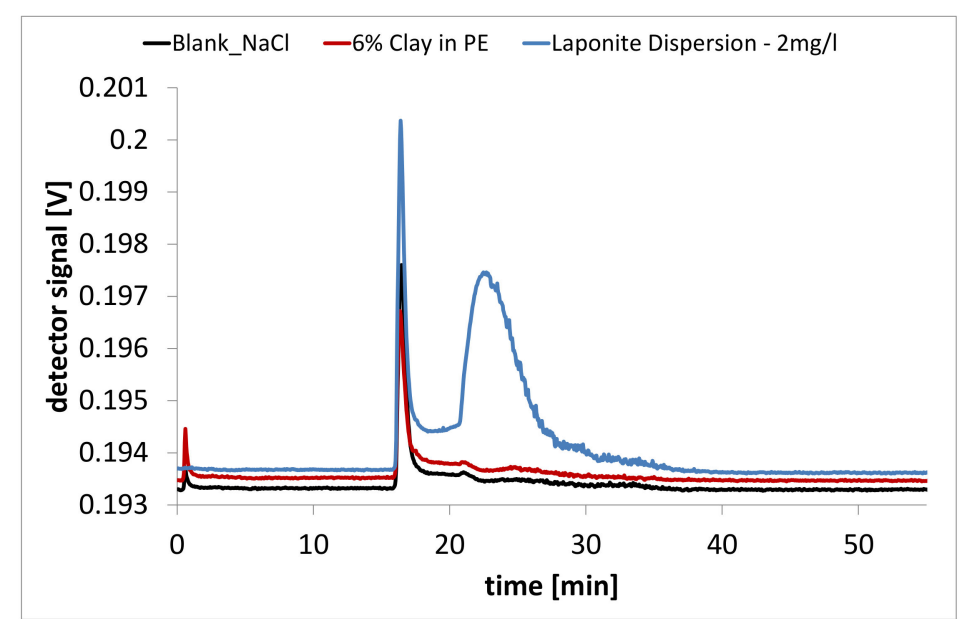

Figure 7. AF4/MALLS fractograms of the abrasion of the LDPE reference without laponite (black), the abrasion of the test sample with $6 \%$ laponite in LDPE (red), and $2 \mathrm{mg} / \mathrm{L}$ laponite dispersion (blue). The red and blue curves were shifted by +0.2 and $+0.4 \mathrm{mV}$, respectively, for better differentiation.

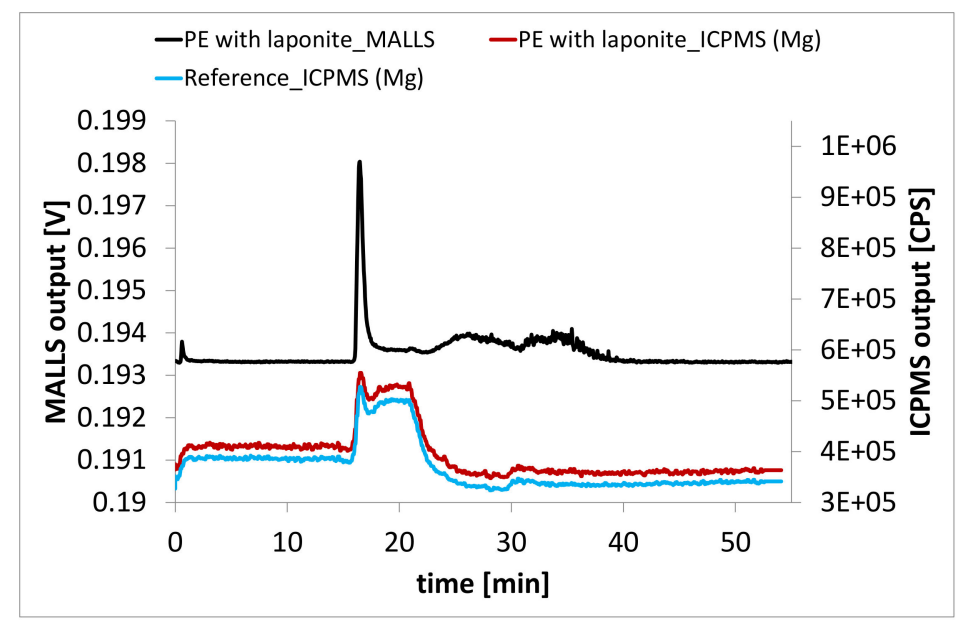

Figure 8. AF4/MALLS/ICP-MS fractograms of a test sample that was swollen first and then abraded: The same sample that caused a signal via MALLS detection (black) at elution times relevant for laponite did not cause a signal for Mg via ICP-MS (online) detection (red). Via AF4/ICP-MS detection no difference between abrasion from test samples and reference samples without laponite (blue, shifted by $-2.5 \times 10^{4}$ CPS for better differentiation) could be found.

Injections of samples prepared from the abrasion of samples that were stretched previously and then abraded by the dry food simulant showed a slight signal via MALLS detection at elution times relevant for laponite particles as well (Figure 9, black fractogram). To differentiate between laponite particles and other contaminants these samples were analyzed by AF4/ICP-MS, too. Again, the identical sample that caused a signal via MALLS detection did not cause a signal for $\mathrm{Mg}$ when measured by AF4/ICP-MS (Figure 9, red fractogram). With a signal in the AF4/MALLS fractogram only, but no signal for Mg in the AF4/ICP-MS fractogram the presence of laponite particles can be excluded. 


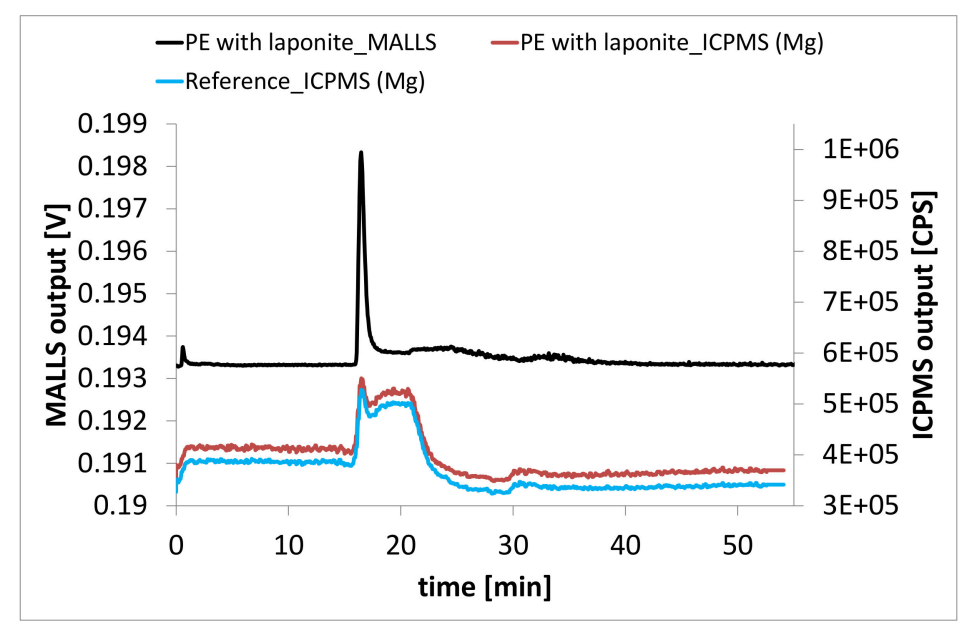

Figure 9. AF4/MALLS/ICP-MS fractograms of a test sample that was stretched first and then abraded: The same sample that caused a signal via MALLS detection (black) at elution times relevant for laponite did not cause a signal for Mg via ICP-MS (online) detection (red). Via AF4/ICP-MS detection no difference between abrasion from test samples and the reference sample withoutlaponite (blue, shifted by $-2.5 \times 10^{4}$ CPS for better differentiation) could be found.

\section{Discussion}

Based on the ICP-MS established LOD for total silver we did not find any detectable silver release from the silver nanocomposite in this study. So, in addition to the previously [1] observed non-migratability of silver nanoparticles under Fickian diffusion conditions, the results of this study support the assumption that also under the foreseeable food contact material stress conditions silver nanoparticles would not be released. When we apply the LOD for total silver of $16 \mathrm{ng} / \mathrm{dm}^{2}$ to the conventional food packaging dimensions of $6 \mathrm{dm}^{2}$ per $1 \mathrm{~kg}$ food-this translates to a detection limit or maximum migration of $0.1 \mu \mathrm{g} / \mathrm{kg}$ food.

Also in case of TiN it is already known that these nanoparticles do not migrate out of the polymer based on Fickian diffusion. It should be noted here that the former migration tests were done in a model study on LDPE samples with an extremely high loading $(1000 \mathrm{mg} / \mathrm{kg})$ of TiN which would not find any application in practice. Therefore, the stress test conditions used in this study can be seen as a worst-case for the release of TiN as well. However, even under the assumption that the detected signals in abrasion tests after additional preceding heating, swelling or stretching of the nanocomposites were solely caused by release of TiN, the released amount of TiN onto the abrasive food simulant would be less than $1 \mu \mathrm{g} / \mathrm{kg}$ (assuming a food packaging with $6 \mathrm{dm}^{2}$ per $\mathrm{kg}$ food and $77.3 \%$ Ti in TiN).

It must be noted that release of Ti was only found in samples that showed impairment caused by the additionally applied prestress conditions. After heating TiN nanocomposites tend to stick together and need to be pulled apart. That way it cannot be excluded that the surface might get slightly damaged. Though swelling in isooctane did not cause migration in our previous migration study, Ti could be found after the nanocomposite was swollen and abraded. Nanocomposites were stickier after storage in isooctane wherefore a higher friction of the dry food simulant can be assumed during the abrasion test. During stretching of the films the film itself becomes thinner and might also be partially damaged (cracks in the surface) whilst TiN particles remain unchanged. Therefore, a direct contact of the simulant with the TiN particles might become possible. Although those stress conditions took obviously influence on the nanocomposites integrity, the test films were not damaged in a way that allows the conclusion that free, unbound TiN particles were abraded from the nanocomposites surface. It seems rather likely that the above-described test scenarios weakened the polymer matrix in a way that allowed abrasion of small polymer flakes in which TiN was still embedded and then measurable by ICP/MS. 
In the case of laponite nanocomposites signals in the AF4/MALLS fractogram, signals were only detected after additionally applied prestress conditions. Since AF4/MALLS does not allow differentiation between laponite and other matrix components in the redispersed abrasion, element-specific ICP-MS measurements were carried out on identical samples. Though magnesium is a ubiquitous element ICP-MS allows sensitive detection of laponite when coupled online to particle-sensitive AF4, because differentiation between magnesium based particles and dissolved magnesium becomes possible. This was demonstrated by comparison of the MALLS signal and the simultaneous recorded ICP-MS signal for an injection of a laponite dispersion into the AF4. At identical elution times ICP-MS gives higher signal intensities for Mg than MALLS does for scattered light (Figure 5). It can be concluded that signal recorded with MALLS but not with ICP-MS cannot be attributed to the presence of laponite. Thus, no release of laponite from any of the mechanical-, thermally-, or chemically-induced stress tests was detected. The signals recorded in the AF4/MALLS fractograms (black curves in Figures 8 and 9) are (again) most likely caused by abraded polymer.

Both, theoretical considerations together with experimental empirical findings demonstrate that nanomaterials are immobilized in a polymer matrix and do not migrate based on Fickian law of diffusion [4,5]. Although it is generally accepted that the limiting factor for migration is the size of the migrant and thus nanomaterials are too large to diffuse, some studies reported positive results in migration experiments from nanocomposites that contained silver [8-11], clays [12-14], or titanium-based nanomaterials like titanium dioxide [15]. The reason for this might be the use of a wrong experimental design. Nanomaterials need both, a setup and analytical techniques that respect the particulate nature of the analyte throughout the experiment. However, it was recently demonstrated that common food simulants used in migration experiments for conventional polymer additives are not necessarily appropriate for nanomaterials, because nanomaterials might show weak stability and the potential to be solubilized. This way release of solubilized nanomaterials might be wrongly (false positive) interpreted as migration of particulate nanomaterials, if only element-specific detection techniques were used (e.g., detection of ionic silver with ICP-MS as a measure for release of silver nanoparticles). Furthermore, the way the samples are prepared is another crucial parameter. Some studies reported that the test samples were cut into small pieces to increase the surface (sample film) to volume (food simulant) ratio. This way the nanocomposite is damaged since at the variety of resulting cutting edges the nanomaterial might not be covered with polymer anymore. That way, no true migration under conditions as applied in practice but release of nanomaterials by dissolution of the nanomaterial itself or its surrounding polymer matrix becomes possible [16,17].

Until now, the number of studies that investigated release of nanomaterials from consumer products after mechanical or otherwise induced stress conditions is limited. In a study of Ntim et al. [18], release of nanomaterials from coated cookware was only detectable after the surface was abraded with a tungsten carbide burr. As the authors stated, this condition obviously damaged the ceramic coating beyond reasonable use. Although the other abrasion conditions with scrubbing pad and steel wool can also be considered as severe, release of nanomaterials was not detected. Göhler et al. [19] found release of particles after abrasion and additionally weathered nanocomposites. However, no release of isolated nanoparticles was found but abrasion of polymer that contained the nanomaterial solely. In another study Golja et al. [20] used an indirect measurement approach based on total Ti (ionic and particulate) without and with ultrafiltration to conclude from the difference on the potential release of particulate $\mathrm{TiO}_{2}$ material. They report a correlation between mechanical stressing and release of nanomaterials [20]. In the respective study scratching of the surface of the pan coating led to higher release levels of titanium dioxide than test samples that were not stressed mechanically. It should be noted that the authors also reported release of $\mathrm{TiO}_{2}$ nanomaterial from unstressed pan coatings, however, using disks punched out of the pan as test specimen with open cut edges and storing them under severe conditions in $3 \%$ acetic acid media. This way, the coating of the pan may have suffered from structural damaging as well. 


\section{Conclusions}

The scope of this study was to explore whether particles of the nanomaterials silver (AgNPs), titanium nitride (TiN), and laponite can be mechanically-released from the food contact surface of polymer-nanocomposites under material stress conditions caused by severe interactions with foods or food simulants including thermal effects as well as solvent based and mechanical stress.

It was demonstrated that in case of abrasion each nanomaterial can be separated from a dry food simulant matrix and redispersed in a suitable solution for subsequent analysis by AF4/MALLS, AF4/MALLS/ICP-MS (laponite), or ICP-MS (AgNPs and TiN) techniques at detection limits of $16.0 \mathrm{ng} / \mathrm{dm}^{2}$ for AgNPs, $39.3 \mathrm{ng} / \mathrm{dm}^{2}$ for TiN, and $12.0 \mu \mathrm{g} / \mathrm{dm}^{2}$ for laponite. Assuming a surface to volume ratio of $6 \mathrm{dm}^{2} / \mathrm{kg}$ of packed food this corresponds to detection limits of approx. $0.1 \mu \mathrm{g} / \mathrm{kg}$ for AgNPs, $0,3 \mu \mathrm{g} / \mathrm{kg}$ for TiN and 71,8 $\mu \mathrm{g} / \mathrm{kg}$ for laponite ( $\mu \mathrm{g}$ nanomaterial per $\mathrm{kg}$ food $(\mathrm{ppb})$ ).

In previous studies it was found and concluded that the nanomaterials investigated in this study cannot migrate based on Fickian diffusion in the polymer. The results of this study confirm that AgNPs and laponite particles are also not released from the nanocomposites' surface under or after severe materials stress conditions. In case of TiN-nanocomposites a release of TiN was detectable at very low levels which were most likely due to abrasion of polymer flakes with incorporated TiN particles and not due to pure TiN particles. In any case, it is most likely that the surface of the composite was severely damaged. However, the measured release of TiN in this case was very low and below $1 \mu \mathrm{g} / \mathrm{kg}$. Furthermore, the used nanocomposite TiN in LDPE at $1000 \mathrm{mg} / \mathrm{kg}$ loading level has the character of a model test sample which will not find application as such in practice.

In conclusion, based on our previous studies and the results of this study, migration, or release of nanoparticles from polymer nanocomposites is very unlikely under usual and even exaggerated conditions of use. Severe and polymer matrix damaging conditions may lead to physical disintegration of the composite which will not necessarily lead to release of the pure nanomaterial but may cause detachment of host matrix particles with embedded nanomaterial as small composite pieces. The crucial prerequisite for non-migration of a nanomaterial from a polymer composite is that it is fully embedded in and covered by the host polymer or matrix.

Author Contributions: Conceptualization, J.B. and R.F.; Methodology, J.B. and R.F.; Validation, R.F., Investigation, J.B.; Writing-Original Draft Preparation, J.B.; Visualization, J.B.; Supervision, R.F.; and Project Administration, R.F.

Acknowledgments: We gratefully acknowledge the financial cosupport of PlasticsEurope and the Cefic FCA (Food Contact Additives) sector group for this study.

Conflicts of Interest: The authors declare no conflicts of interest.

\section{References}

1. Bott, J.; Störmer, A.; Franz, R. A Comprehensive Study into the Migration Potential of Nano Silver Particles from Food Contact Polyolefins, in Chemistry of Food, Food Supplements, and Food Contact Materials: From Production to Plate; Benvenuto, M.A., Ed.; American Chemical Society: Washington, DC, USA, 2014; pp. 51-70.

2. Bott, J.; Störmer, A.; Franz, R. A model study into the migration potential of nanoparticles from plastics nanocomposites for food contact. Food Packag. Shelf Life 2014, 2, 73-80. [CrossRef]

3. Bott, J.; Franz, R. Investigation into the Potential Migration of Nanoparticles from Laponite-Polymer Nanocomposites. Nanomaterials 2018, 8, 723. [CrossRef] [PubMed]

4. Franz, R.; Welle, F. Mathematic modelling of migration of nanoparticles from food contact polymers. In The Use of Nanomaterials in Food Contact Materials-Design, Application, Safety; Veraart, R., Ed.; DEStech Publications Inc.: Lancaster, PA, USA, 2017.

5. Störmer, A.; Bott, J.; Kemmer, D.; Franz, R. Critical review of the migration potential of nanoparticles in food contact plastics. Trends Food Sci. Technol. 2017, 63, 39-50. [CrossRef]

6. Cavallaro, G.; Lazzara, G.; Milioto, S. Dispersions of Nanoclays of Different Shapes into Aqueous and Solid Biopolymeric Matrices. Extended Physicochemical Study. Langmuir 2011, 27, 1158-1167. [PubMed] 
7. Cavallaro, G.; Lazzara, G.; Milioto, S. Aqueous phase/nanoparticles interface: Hydroxypropyl cellulose adsorption and desorption triggered by temperature and inorganic salts. Soft Matter 2012, 8, 3627-3633. [CrossRef]

8. Artiaga, G.; Ramos, K.; Ramos, L.; Cámara, C.; Gómez-Gómez, M. Migration and characterisation of nanosilver from food containers by AF(4)-ICP-MS. Food Chem. 2015, 166, 76-85. [CrossRef] [PubMed]

9. Huang, Y.; Chen, S.; Bing, X.; Gao, C.; Wang, T.; Yuan, B. Nanosilver Migrated into Food-Simulating Solutions from Commercially Available Food Fresh Containers. Packag. Technol. Sci. 2011, 24, 291-297. [CrossRef]

10. Song, H.; Li, B.; Lin, Q.B.; Wu, H.J.; Chen, Y. Migration of silver from nanosilver-polyethylene composite packaging into food simulants. Food Addit. Contam. Part A 2011, 28, 1758-1762. [CrossRef] [PubMed]

11. Von Goetz, N.; Fabricius, L.; Glaus, R.; Weitbrecht, V.; Gunther, D.; Hungerbuhler, K. Migration of silver from commercial plastic food containers and implications for consumer exposure assessment. Food Addit. Contam. Part A 2013, 30, 612-620. [CrossRef] [PubMed]

12. Echegoyen, Y.; Rodriguez, S.; Nerin, C. Nanoclay migration from food packaging materials. Food Addit. Contam. Part A 2016, 33, 530-539. [CrossRef] [PubMed]

13. Farhoodi, M.; Mousavi, S.M.; Sotudeh-Gharebagh, R.; Emam-Djomeh, Z.; Oromiehie, A. Migration of Aluminum and Silicon from PET/Clay Nanocomposite Bottles into Acidic Food Simulant. Packag. Technol. Sci. 2013, 27, 161-168. [CrossRef]

14. Schmidt, B.; Katiyar, V.; Plackett, D.; Larsen, E.H.; Gerds, N.; Koch, C.B.; Petersen, J.H. Migration of nanosized layered double hydroxide platelets from polylactide nanocomposite films. Food Addit. Contam. Part A 2011, 28, 956-966. [CrossRef] [PubMed]

15. Lin, Q.B.; Li, H.; Zhong, H.N.; Zhao, Q.; Xiao, D.H.; Wang, Z.W. Migration of Ti from nano-TiO-polyethylene composite packaging into food simulants. Food Addit. Contam. Part A 2014, 31, 1284-1290.

16. Duncan, T.V. Release of engineered nanomaterials from polymer nanocomposites: The effect of matrix degradation. ACS Appl. Mater. Interfaces 2015, 7, 20-39. [CrossRef] [PubMed]

17. Duncan, T.V.; Pillai, K. Release of Engineered Nanomaterials from Polymer Nanocomposites: Diffusion, Dissolution, and Desorption. ACS Appl. Mater. Interfaces 2015, 7, 2-19. [CrossRef] [PubMed]

18. Addo Ntim, S.; Norris, S.; Scott, K.; Thomas, T.A.; Noonan, G.O. Consumer use effects on nanoparticle release from commercially available ceramic cookware. Food Control 2018, 87, 31-39. [CrossRef]

19. Göhler, D.; Nogowski, A.; Fiala, P.; Stintz, M. Nanoparticle release from nanocomposites due to mechanical treatment at two stages of the life-cycle. J. Phys. Conf. Ser. 2013, 429, 012045. [CrossRef]

20. Golja, V.; Dražić, G.; Lorenzetti, M.; Vidmar, J.; Ščančar, J.; Zalaznik, M.; Kalin, M.; Novak, S. Characterisation of food contact non-stick coatings containing TiO2 nanoparticles and study of their possible release into food. Food Addit. Contam. Part A 2017, 34, 421-433. [CrossRef] [PubMed]

(C) 2019 by the authors. Licensee MDPI, Basel, Switzerland. This article is an open access article distributed under the terms and conditions of the Creative Commons Attribution (CC BY) license (http://creativecommons.org/licenses/by/4.0/). 